\title{
With Antibiotic Cement Versus Without Antibiotic Cement in the Treatment of Tibial Osteomyelitis After Radical Debridement: A Randomized Controlled Trial
}

Jinxing Yang ( $\square$ yangjx1977@126.com )

Department of Orthopaedic Trauma Shenzhen Second People's Hospital/First Affiliated Hospital of Shenzhen University Health Science Center No. 3002, Sungang West Road Shenzhen, China https://orcid.org/0000-0002-1880-0706

Yun Han

Shenzhen Second People's Hospital

Lijun Liu

Shenzhen Second People's Hospital

Hongxian Chen

Shenzhen Second People's Hospital

Junfeng Huang

Shenzhen Second People's Hospital

$\mathrm{Xu} \mathrm{Xu}$

Shenzhen Second People's Hospital

Research article

Keywords: antibiotic cement, osteomyelitis, Cierny-Mader Type IV tibial osteomyelitis

Posted Date: September 8th, 2020

DOI: https://doi.org/10.21203/rs.3.rs-38844/v1

License: (c) (i) This work is licensed under a Creative Commons Attribution 4.0 International License. Read Full License 


\section{Abstract}

Background: In most of the cases, antibiotic cement is recommended to fill the dead space after debridement of osteomyelitis. As to local Cierny-Mader Type IV tibial osteomyelitis, radical debridement of the infected bone can be achieved, after that, is it still necessary to apply antibiotic cement to the dead space? This study was aimed to answer the question.

Methods: From January 2010 to January 2015, 64 patients with local Cierny-Mader Type IV tibial osteomyelitis were included and randomly divided into two groups. Patients in the cement group were treated with antibiotic-impregnated bone cement after radical debridement of the infected bone (cement group), while the other patients in the without cement group were not applied any cement after the same debridement (without cement group). Both groups were operated by the same surgical team and applied llizarov bone transport to fill the bone defect.

Result: All patients in both groups achieved successful healing with bone union at both corticotomy and tibial docking sites. At the end of follow-up there was one case of infection recurrence in each groups. There were no differences in demographics and clinical variables between the two groups. There was also no difference either in terms of bone results or functional results between the two groups $\left(c^{2}=0.006\right.$ for bone results and $c^{2}=1.339$ for functional results). But there was significant difference in terms of total hospitalization expenses between the two groups ( $\mathrm{\otimes} \otimes 0.001$, the cement group was much higher than the without cement group).

Conclusion: When radical debridement is achieved in dealing with local Cierny-Mader Type IV tibial osteomyelitis, it is no need to apply antibiotics cement in the dead space, but it needs more cases to increase the convincingness.

Trial registration: ChiCTR-TNRC-10002837Øretrospectively registered.

\section{Background}

Osteomyelitis represents one of the most challenging diseases in orthopedic surgery. It consists of a destructive inflammatory process in the bone caused by infectious microorganisms ${ }^{[1]}$. The high recurrence rate gives it a bad reputation as "bone cancer". Over the past 20 years, the treatment strategy for osteomyelitis has changed to a great extent because of better staging systems, advanced surgical techniques, antibiotic regimens, and adjuvant treatment modalities ${ }^{[2]}$. Cierny et al. ${ }^{[3]}$ classified chronic osteomyelitis into four anatomic types and further staged the pathology according to the extent of local and systemic compromise in patients. As for the treatment of Cierny-Mader Type IV osteomyelitis, the most popular consensus in recent years is radical debridement of infected bone, which results in a long bone defect ${ }^{[4-5]}$. Several techniques ${ }^{[6-13]}$ are recommended to reconstruct the long bone defects.

Vascularized fibular graft ${ }^{[6]}$ can be extremely difficult to apply. In addition, mismatch between tibial and fibular cross-sectional areas may result in a high rate of refracture. These factors limit application of 
vascularized fibular graft in the clinic. Recently, Masquelet ${ }^{[7-9]}$ proposed a method combining cancellous bone autografts and induced membranes for the management of segmental bone defects. The shortage of this technique is difficult to get enough cancellous bone and the quality of the bone healing is controversial. Over the years, llizarov bone transport is recommended by more and more doctors ${ }^{[10-13]}$. It can provide a large diameter of bone with intact blood supply to fill the bone defect. Moreover, this method has the ability to correct coinciding deformity, shortening, soft tissue loss, and nearby joint contractures.

After resection of infected bone, the common guideline suggests to fill the dead space with antibiotic material to help eliminating infection. In early days, the popular

antibiotic material is antibiotic-impregnated bone cement $(\mathrm{PMMA})^{[14-17]}$, which is temporary use and needs extra procedure to remove it in around six weeks after operation. With the development of synthetic bone substitutes, many new materials are absorbable, such as calcium sulphate (CS) and bioglass. But these materials are either very expensive or related to wound issues owing to serous exudate ${ }^{[18-21]}$. Since radical debridement has completely destroyed the protective barriers for bacterial, there is no place for bacterial to hide from the attack of intravenous antibiotics, is antibiotic material still needed in the dead space邓

In this randomized controlled trial, 64 patients with local Cierny-Mader Type IV tibial osteomyelitis were randomly divided into two groups: one group were treated with antibiotic-impregnated bone cement after resection of infected bone, another group were not after resection of infected bone. Both groups were applied llizarov bone transport to fill the bone defect. We compared the clinical outcome of the two groups, and sought to find out the necessity of applying antibiotic-impregnated materials in the treatment of local Cierny-Mader Type IV tibial osteomyelitis after radical debridement.

\section{Methods}

This study is a pragmatic single centered randomized control trial (Fig. 1), which took place at our orthopaedic trauma center (Level One Trauma Centers) in Shenzhen, China, from 2010 to 2015. Informed consent was obtained from each individual participant and Ethics Committee of clinical scientific research of the First Affiliated Hospital of Shenzhen University approved this study. Those who do not have any mental disorders were enrolled, and all of them signed the informed consent form in a clearconscious situation without any temptation. There are no conflict of interests for any of the authors with regard to this study.

\section{Sample Size Calculation:}

The primary outcome measure was the rate of infection recurrence. Using an effect size of approximately $10 \%$ and a variance of $5 \%$, it was calculated that about 30 patients per group would be needed to obtain $80 \%$ power with a single side alpha level of 0.05 . Accounting for a $4 \%$ drop out rate, we planned to recruit 32 patients per group, for a total of 64 patients in the trial. 


\section{Inclusion Criteria}

Patients with local Cierny-Mader Type IV tibial osteomyelitis were included.

\section{Exclusion Criteria}

Exclusion criteria included patient and osteomyelitis specific factors.

Patient-related exclusion factors included:

1. Medical comorbidity precluding operative intervention

2. Inability to comply with postoperative protocol (ie, advanced dementia)

3. Refusal to carry an external fixator

Osteomyelitis -related exclusion factors included:

1. Osteomyelitis extended to distal/proximal tibial epiphysis which could not be performed radical debridement due to salvage the adjacent joints

2. Other types of tibial osteomyelitis except local Cierny-Mader Type IV

There were no exclusion criteria with regard to patient age, presence of osteoporosis/osteopenia, or body mass index (BMI).

\section{Randomization}

Randomization was performed by the research coordinators at admission by use of concealed sequentially numbered, opaque, sealed envelopes, in 1:1 ratio. Patients were randomized to 1 of 2 treatment groups: (1) "cement group", or (2) "without cement group". Allocation was only concealed from patients and it was a single blind study.

\section{Treatment}

Preoperative examination included radiography, magnetic resonance imaging and laboratory examination (Fig. 2). Sinus tract tissues and deep wound drainage must be sent to culture the pathogenic bacteria and identified the sensitive antibiotics. For the cases without sinus, biopsy needed to be done. Systemic sensitive antibiotics were applied to control the acute inflammation before surgery. Some internal diseases, such as anemia, hypoproteinemia, and diabetes mellitus, also must be treated before surgery. According to physical and imaging examinations, extent of bone resection and external fixator was preliminarily determined preoperatively.

All patients were operated without use of a tourniquet, so as to differentiate viable soft tissue and bone. After the removal of hard wares $囚$ if there existed $囚$, we resected infected bone along the normal tibial bone. Cortical bleeding, the so-called paprika sign must be seen during the operation (Fig. 3). Inflammatory pseudomembranes, necrosis tissues and abscess must be debrided thoroughly, but inflammatory skin 
could be reserved. Due to the reservation of inflammatory skin and resection of the infected bone, most of the cases could close the wound, only 1 case in without cement group needed an extra local flap to cover the wound. Infected bone and soft tissue cultures were taken to ascertain the appropriate antibiotic treatment in the postoperative period.

After complete hemostasis of the wound, the dead space was filled with custom-made antibioticimpregnated cement $(2.4 \mathrm{~g}$ teicoplanin and $40 \mathrm{~g}$ polymethylmethacrylate powder in bead form, the trade name of the cement was Palacons Bone Cement, manufactured by Waldemar Link GmbH \& Co.KG ) in cement group. In without cement group, only a drainage tube was put into the dead space (Fig. 4). Orthofix LRS(Limb Reconstruction System) external fixators were performed in most of the cases, only 1 case in cement group was fixed with llizarov ring frame due to the distal part of the tibial was too short after infected bone resection, and 1 case in without cement group was fixed with llizarov ring frame for the same reason.

One week later, tibial osteotomy in both groups were carried on for bone transport. Through a separate mini-incision, corticotomy of health bone was performed at the distal or proximal tibial metaphysis, depending on the bone defect site.

Systemic antibiogram-specific antibiotics were continued for approximately 6 weeks after the first operation according to intraoperative cultures and sensitivity.

Bone transport started on the 7th day after tibial corticotomy in both group, and proceeded at a initial rate of $1 \mathrm{~mm}$ per day, which was divided into 4 times of $0.25 \mathrm{~mm}$ each. During hospitalization, patients were trained to distract the external fixator. On the second day after surgery, physical therapy was started included progressively partial weight bearing with crutches, knee and ankle range of movement exercises, and isometric and isotonic muscle strengthening exercises.

\section{Follow-up}

Radiographic evaluation was performed every two weeks during the distraction phase and every four weeks during the consolidation phase. Radiographies were ordered to monitor callus growth in the distraction areas and alignment of the transport segment. Distraction rate was adjusted according to $x$ ray controls. The antibiotic cement beads in cement group were withdrawn at about 6-8 weeks after first operation, which depended on whether the cement beads block the bone transport. Once docking was achieved, docking site grafting ( cancellous bone from iliac crest) was performed in most of patients (Fig. 5 ), there were only 2 patients in each group without docking site grafting because of good contact around the docking sites. Simultaneous adjustment of bone transport segment was performed if the bone transport segment lost its alignment. Before stopping bone distraction, a full length lower limb X-ray was order to check leg length discrepancy (LLD). If LLD exist, limb lengthening was performed until the length of both lower limb were equal. External fixator was not removed until consolidation of the distraction area and union of the docking site were achieved (Fig.6). 


\section{Outcome Measures}

The primary outcome measure was rate of infection recurrence. Secondary outcome measures included: bone results and functional results, rate of complications, external fixator index and total hospitalization expenses.

Bone and functional outcomes were evaluated by using Paley's scoring system ${ }^{[22,23]}$. The bone scoring system was based on five criteria: union, infection, deformity, LLD, and the cross-sectional area of union of the regenerate bone and docking site, and graded as excellent, good, fair and poor. The functional scoring system was also based on five criteria: pain; need for walking aids or braces; foot, ankle, or knee deformity or contracture; ankle and/or subtalar loss of range of motion as compared with the preoperative range; and ability to return to normal activities of daily living and/or work, and also graded as excellent, good, fair and poor. Complications were recorded, which were grouped as minor, major without residual sequelae, and major with residual sequelae ${ }^{[23]}$.

\section{Data Analysis}

Bone and functional scores were evaluated with Chi square test of rowxlist. The Mann-Whitney U test was used for statistical analysis to evaluate significant differences between two groups in demographics, number of complications per patient, External fixator index and total hospitalization expenses. The significance threshold was $p<0.05$ and the analyses were two-way. Analyses were done using SPSS 23 software.

\section{Results}

From January 2010 to January 2015, 64 patients with local Cierny-Mader Type IV tibial osteomyelitis at our institution were initially enrolled and randomly divided into two groups(cement group and without cement group). Among them, 5 patients lost follow-up, the rest of patients (59 patients) were finally included: 30 patients in the cement group and 29 patients in without cement group.

In cement group, there were 19 males and 11 females, with a mean age of 40.6 years (range,17-66 years). According to Cierny-Mader patient classification, 3 patient belonged to type $A$ and 27 patients belonged to type B (26 patients with sinus tract, 3 patient with diabetes, 5 patient with hypertension and coronary disease). The infection source of osteomyelitis: 25 patients were post-traumatic, 5 patients were hematogenous. The mean bone loss after infected bone resection was $7.6 \mathrm{~cm}($ range, $4.8-13.0 \mathrm{~cm})$.

In without cement group, there were 20 males and 9 females, with a mean age of 40.5 years (range,17-70 years). According to Cierny-Mader patient classification, 2 patient belonged to type $A$ and 27 patients belonged to type $B$ ( 26 patients with sinus tract, 3 patients with diabetes, 4 patient with hypertension and coronary disease). The infection source of osteomyelitis: 24 patients were post-traumatic, 4 patients were hematogenous and 1 patient were from local. The mean bone loss after infected bone resection was $7.6 \mathrm{~cm}$ (range, $4.5-12.8 \mathrm{~cm})$. 
There were no differences in demographics and clinical variables between the two groups (Table 1). The mean follow-up was not statistically different between two groups, 71.7 months (range, 60-86 months) in cement group and 71.4 months (range, 60-85) in without cement group ( $p=0.94$, Table 2 ).

All patients in both groups achieved successful healing with bone union at both corticotomy and tibial docking sites. At the end of follow-up囚each group had one case of infection recurrence. There was no difference either in terms of bone results or functional results between the two groups $\left(c^{2}=0.006\right.$ for bone results and $c^{2}=1.339$ for functional results, Table 2 ). In the cement group, the bone results were excellent in 24 patients, good in 6 . The functional results were excellent in 22 patients, good in 4, and fair in 2 . In the without cement group, bone results were excellent in 24 patients, good in 5 . The functional results were excellent in 24 patients, good in 4 and fair in 1.

The external fixation index (EFI) were no significant differences between two groups $(p=0.905$, Table 3$)$. In cement group, the mean EFI was 49.0 days/ cm. In without cement group, the mean EFI was 49.5 days/ $\mathrm{cm}$.

Due to distraction rate control, early physical therapy, aggressive docking side grafting and adjustment of docking malalignment, there were no residual sequelae in both groups. The number of complications per patient (Table 3) was no significant differences between two groups (1.15 vs 1.07 in cement group and without cement group, respectively; $p=0.34$, Table 3 ). The major complications were pin-tract infection, 23 cases in cement group and 22 cases in without cement group. The other complications were docking malalignment ( 6 cases in cement group and 6 cases in without cement group), skin invagination (4 cases in cement group and 5 cases in without cement group) and limited range of joint motion (4 cases in cement group and 4 cases in without cement group).

Total hospitalization expenses in cement group were $28.7 \%$ higher than that in without cement group, there were significant differences between two groups (70954.1 vs 55105.2 in cement group and without

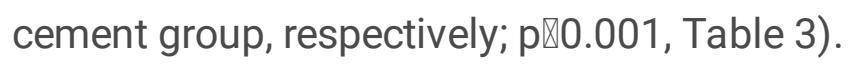

\section{Discussion}

Osteomyelitis is an infection of the bone. The high recurrence rate continues to pose a major challenge to orthopaedic surgeons ${ }^{[25]}$. The Mayo Clinic reported a $20 \%$ failure rate in the management of chronic infections ${ }^{[26]}$. Usually after the bone is infected, Leukocytes are attracted to the area and secrete enzymes in attempt to kill the bacteria. Blood flow to the area is decreased and a devitalized, necrotic bone is formed which called a sequestrum. The sequestrum is an infected, dead bone resulting from osteomyelitis. The sequestrum has no connection to normal bone through the haversian system, Because the sequestrum is avascular or a dead piece of bone. Antibiotics can not reach the sequestrum, which makes it to be the optimal place to protect the bacteria against the attack from antibiotics. Aggressive infected bone resection is an osteoctomy along the normal adjacent bone. The whole infected bone is removed and no sequestrum is left for the bacteria to stay. 
Ilizarov bone transport technique is widely recommended to fill the gap after the infected bone resection ${ }^{[2,12,23]}$. It can provide a symmetrical bone with intact blood supply to fill the bone defect. Moreover, this method also can simultaneously correct coinciding deformity, shortening, soft tissue loss, and nearby joint contractures. During the bone transport, the distraction not only regenerate bone tissue, but also regenerate vessels, which will increase the blood supply to the area and help to eradicate the bacteria.

It is difficult to manage a radical debridement for osteomyelitis. Cierny-Mader Type I osteomyelitis is hard to get complete debridement because of the irregular medullary cavity, many small or tiny sequestrums are still remain after the reaming debridement. It is the same for some Cierny-Mader Type IV osteomyelitis which infection extend to the adjacent joints. When trying to salvage the joints, the debridement is not thorough. For the above situation, antibiotic material is necessary to help to eradicate the bacteria. But when we achieve a radical debridement for local Cierny-Mader Type IV tibial osteomyelitis, there is no sequestrum left for the bacteria to hide against the attack of antibiotics and the increasing blood supply during the distraction will provide extra help to eliminate the bacteria, is antibiotic material still needed in the dead space区

This randomised controlled trial was aimed to answer this question. Current consensus takes radical debridement as the key point in the treatment of osteomyelitis. All the debridement in this study were needed to match the following two criteria: infected bone resection along the normal adjacent bone and thorough debridement of Inflammatory pseudomembranes, necrosis tissues and abscess. The recurrence of infection is the major concern in this study, only patients who had been followed up for at least five years were included, which is better to tell the story.

In this study, all the patient had been followed up not less than five years: 71.7 months (range, 60-86 months) in cement group and 71.4 months (range, 60-85) in without cement group. There was not statistically different between two groups ( $p=0.94$, Table 3 ). There were also no differences in demographics and clinical variables (age, bone loss after resection) between the two groups (Table 1). Above circumstances makes the comparison between the two groups is meaningful.

At the end of the follow-up, each group had one case of infection recurrence, the recurrence rate was almost no difference (3.33\% in cement group VS 3.45\% in without cement group). This indicates no benefits to apply antibiotic material in the dead space after radical debridement. After the resection of infected bone, it was easy to perform thorough debridement of Inflammatory pseudomembranes, necrosis tissues and abscess. When above radical debridement was done, there was only some bacteria remain in the soft tissue which can be easily eradicated by the intravenous sensitive antibiotics. This is the same in the treatment of cellulitis, sensitive antibiotics is enough to achieve a good result.

Due to the use of antibiotic cement and the extra surgery to withdraw the cement, total hospitalization expenses in cement group were much higher than that in without cement group. The average hospitalization expenses in cement group increased $28.7 \%$ as contrast to the without cement group. 
Patients suffering from osteomyelitis usually have been through many operations, their economic situation is poor. The additional hospitalization costs will add a heavy burden to them.

Some articles show the antibiotic cement can create a soft tissue tunnel for bone transport, induce a pseudosynovial membrane, prevent fibrous tissue interposed at the docking side and secret bone morphogenetic protein, hence, it can increase the possibility of spontaneous docking side union. But this didn't happen in our cases. Two patients in each group achieved spontaneous docking side union were because the bone transport segments embedded into the distal or proximal tibial metaphysis. As the patients were performed docking side grafting, interposed soft tissue and poor contact could been saw in each case. The major problems of the docking side healing are poor contact and sclerosis among the docking side, antibiotic cement can't do any help.

There was no difference between the two techniques in terms of the bone result and the functional result. There was also no difference between the two groups in terms of the number of complications per patient. These suggest that with or without antibiotic cement is the same result for the treatment of local Cierny-Mader Type IV osteomyelitis after radical debridement. In our cases, the number of complications per patient is lesser than many other literatures ${ }^{[10,11,23]}$, this is because we took the docking side grafting as a routine procedure when the contact of the docking side was poor.

In our cases, only 1 case in without cement group needed an extra local flap to cover the wound. This was not only because there were no large skin defect in our cases, but also due to we performed lesser aggressive debridement to the soft tissue than to the bone. When performing soft tissue debridement, we remained the inflammatory skin after thorough debridement of Inflammatory pseudomembranes, necrosis tissues and abscess. With the absence of the infected bone, most of the wounds could be closed. The infection of inflammatory skin could be treated by the intravenous sensitive antibiotics and this method was proved to be safe in this study.

\section{Conclusions}

In conclusion, when radical debridement as follows are achieved: infected bone resection along the normal adjacent bone and thorough debridement of inflammatory pseudomembranes, necrosis tissues and abscess, it is no need to apply antibiotics cement in the dead space when dealing with local CiernyMader Type IV tibial osteomyelitis, but it needs more cases to increase the convincingness.

\section{List Of Abbreviations}

CS calcium sulphate

BMI body mass index

LRS limb Reconstruction System 
LLD leg length discrepancy

EFI external fixation index

\section{Declarations}

\section{Ethics approval and consent to participate}

Informed consent was obtained from each individual participant and Ethics Committee of clinical scientific research of the First Affiliated Hospital of Shenzhen University approved this study. Those who do not have any mental disorders were enrolled, and all of them signed the informed consent form in a clear- conscious situation without any temptation.

\section{Consent for publication}

All authors have approved the version submitted for publication.

\section{Availability of data and material}

The detailed data of the patients are stored in the medical record room of our hospital (Shenzhen Second People's Hospital/First Affiliated Hospital of Shenzhen University Health Science Center, Shenzhen, 518035, China) and can be obtained by calling 0086-0755-83366388-8016.

\section{Competing interests}

The authors declare that they have no conflicts of interest.

\section{Funding}

This study was supported by the Key Specialized Promotion Project of Shenzhen Health Bureau (SZXJ2018054).

\section{Authors' contributions}

JXY contributed to the conception of the study, performed the operations, managed the patients and wrote the manuscript;

LJL contributed to the conception of the study;

YH performed the enrollment and follow-up of the patients;

HXC helped perform the operations;

JFH helped perform the operations;

XX performed the data analyses and manuscript preparation. 
Acknowledgements

My gratitude goes deeply to Professor Lijun Liu, my supervisor, for his constant encouragement and guidance. He has walked me through all the stages of this clinial trial. Without his consistent and illuminating instruction, this trial could not be finished successfully.

\section{References}

[1] Romano CL, Romano D, Logoluso N, Drago L. Bone and joint infections in adults: a comprehensive classification proposal. Eur Orthop Traumatol 2011; 1: 207-217.

[2] Cierny G 3rd. Infected tibial non-union (1981- 1995): the evolution of change. Clin Orthop Relat Res.1999; 360:97-105.

[3] Cierny G 3rd, Mader JT, Penninck JJ. A clinical staging system for adult osteomyelitis. Clin Orthop Relat Res. 2003; 414:7-24.

[4] Lew DP, Waldvogel FA. Osteomyelitis. Lancet (London, England) 2004; 364: 369-379.

[5] Haas DW, McAndrew MP. Bacterial osteomyelitis in adults: evolving considerations in diagnosis and treatment. Am J Med 1996; 101: 550-561.

[6] Pederson WC, Person DW. Long bone reconstruction with vascularized bone grafts. Orthop Clin North Am. 2007;38(1):23-35.

[6] Masquelet AC. Muscle reconstruction in reconstructive surgery: soft tissue repair and long bone reconstruction. Langenbecks Arch Surg. 2003; 388(5): 344-346.

[7] Masquelet AC, Begue T. The concept of induced membrane for reconstruction of long bone defects. Orthop Clin North Am. 2010;41(1):27-37.

[8] Biau DJ, Pannier S, Masquelet AC, Glorion C. Case report: reconstruction of a 16-cm diaphyseal defect after Ewing's resection in a child. Clin Orthop Relat Res. 2009;467(2):572-7.

[9] Dendrinos GK, Kontos S, Lyritsis E. Use of the llizarov technique for treatment of non-union of the tibia associated with infection. J Bone Joint Surg Am. 1995; 77:835-846.

[10] Green SA, Jackson JM, Wall DM, Marinow H, Ishkanian J. Management of segmental defects by the llizarov intercalary bone transport method. Clin Orthop Relat Res. 1992; 280:136142.

[11] Liu T, Zhang X, Li Z, Peng D. Management of combined bone defect and limb length discrepancy after tibial chronic osteomyelitis. Orthopaedics. 2011; 34: e363-e367.

[12] Rozbruch SR, Weitzman AM, Watson JT, Freudigman P, Katz HV, llizarov S. Simultaneous treatment of tibial bone and soft-tissue defects with the llizarov method. J Orthop Trauma.2006;20:197-205. 
[13] Blaha JD, Calhoun JH, Nelson CL, Henry SL, Seligson D, Esterhai JL Jr, et al. Comparison of the clinical efficacy and tolerance of gentamicin PMMA beads on surgical wire versus combined and systemic therapy for osteomyelitis. Clin Orthop Relat Res. 1993;295:8-12.

[14] Calhoun JH, Henry SL, Anger DM, Cobos JA, Mader JT. The treatment of infected nonunions with gentamicin-polymethylmethacrylate antibiotic beads. Clin Orthop Relat Res. 1993;295:23-7.

[15] Walenkamp GH, Kleijn LL, de Leeuw M. Osteomyelitis treated with gentamicinPMMA beads: 100 patients followed for 1-12 years. Acta Orthop Scand. 1998;69(5):518-22.

[16] Mohanty SP, Kumar MN, Murthy NS. Use of antibiotic-loaded polymethyl methacrylate beads in the management of musculoskeletal sepsis-a retrospective study. J Orthop Surg (Hong Kong). 2003;11(1):73-9.

[17] Ferguson JY, Dudareva M, Riley ND, Stubbs D, Atkins BL, McNally MA. The use of a biodegradable antibiotic-loaded calcium sulphate carrier containing tobramycin for the treatment of chronic osteomyelitis: a series of 195 cases. Bone Joint J 2014; 96B: 829-836.

[18] McKee MD, Wild LM, Schemitsch EH, Waddell JP. The use of an antibiotic-impregnated, osteoconductive, bioabsorbable bone substitute in the treatment of infected long bone defects: early results of a prospective trial. J Orthop Trauma 2002; 16: 622-627.

[19] McAndrew J, Efrimescu C, Sheehan E, Niall D. Through the looking glass; Bioactive glass S53P4 (BonAliveß) in the treatment of chronic osteomyelitis. Ir J Med Sci 2013; 182: 509-511.

[20] Aurégan J-C, Bégué T. Bioactive glass for long bone infection: a systematic review. Injury 2015; 46: S3-S7.

[21] Fischgrund J, Paley D, Suter C. Variables affecting time to bone healing during limb lengthening. Clin Orthop 1994;301:31-37.

[22] Paley D, Catagni MA, Argnani F, Villa A, Benedetti GB, Cattaneo R. Ilizarov treatment of tibial nonunions with bone loss. Clin Orthop 1989;241:146-165.

[23] Paley D, Maar DC. Ilizarov bone transport treatment for tibial defects. J Orthop Trauma. 2000;14:76-85.

[24] Lazzarini L, Mader JT, Calhoun JH (2004) Osteomyelitisin Long Bones. J Bone Jt Surg Am 86(10):2305-2318.

[25] Hall BB, Fitzgerald RH, Rosenblatt JE (1984) Anaerobic osteomyelitis. J Bone Jt Surg Am 65:30-35.

\section{Tables}


TABLE 1: Demographic data of the two groups ( ${ }^{\star}$ Analyzed by Mann-Whitney U test)

\begin{tabular}{|lllc|}
\hline Variable & $\begin{array}{l}\text { Cement group } \\
(\mathrm{N}=30)\end{array}$ & Without cement group $(\mathrm{N}=29)$ & $\mathrm{P}$ value* \\
\hline Mean age(years) & $40.6($ range 33-66) & 40.5(range 17-70) & $\mathrm{P}=0.98$ \\
\hline Mean bone loss after resection(cm) & $7.6($ range 5.2-13.0 & $7.6($ range 4.5-12.8) & $\mathrm{P}=0.83$ \\
& ) & & \\
\hline Mean follow up(months) & $71.7($ range 60-86) & $71.4($ range 60-85) & $\mathrm{P}=0.94$ \\
\hline
\end{tabular}

TABLE 2: Comparison of outcome parameters between the two groups ( ${ }^{\star}$ Analyzed by Chi square test of row $\times$ list.)

\begin{tabular}{|llll|}
\hline Parameter & $\begin{array}{l}\text { Cement group } \\
(\mathrm{N}=30)\end{array}$ & Without cement group $(\mathrm{N}=29)$ & $\mathrm{c}^{2}$ value* \\
\hline Bone results & 24 excellent, 6 good & 24 excellent, 5 good & $\mathrm{c}^{2}=0.006$ \\
\hline Funtional results & 24 excellent, 4 good, 2 fair & 24 excellent, 4 good, 1 fair & $\mathrm{c}^{2}=1.339$ \\
\hline
\end{tabular}

TABLE 3: Comparison of outcome parameters between the two groups ( ${ }^{\star}$ Analyzed by Mann-Whitney U test.)

\begin{tabular}{|c|c|c|c|}
\hline Parameter & $\begin{array}{l}\text { Cement group } \\
(\mathrm{N}=30)\end{array}$ & $\begin{array}{l}\text { Without cement } \\
\text { group }(\mathrm{N}=29)\end{array}$ & $P$ value* \\
\hline External fixator index(days/cm) & 49.0(range 35.7-60.3) & 49.5(range 36.7-66.4) & $P=0.905$ \\
\hline $\begin{array}{l}\text { Number of } \\
\text { complications/patient (Mean) }\end{array}$ & 1.15 (range $0-3$ ) & 1.07(range $0-3$ ) & $P=0.34$ \\
\hline $\begin{array}{l}\text { Total cost of hospitalization } \\
\text { (yuan) }\end{array}$ & $\begin{array}{l}\text { 70954.1(range 62994.2- } \\
82312.8 \text { ) }\end{array}$ & $\begin{array}{l}\text { 55105.2(range 49982.8- } \\
\text { 71003.5) }\end{array}$ & $P \bowtie 0.001$ \\
\hline
\end{tabular}




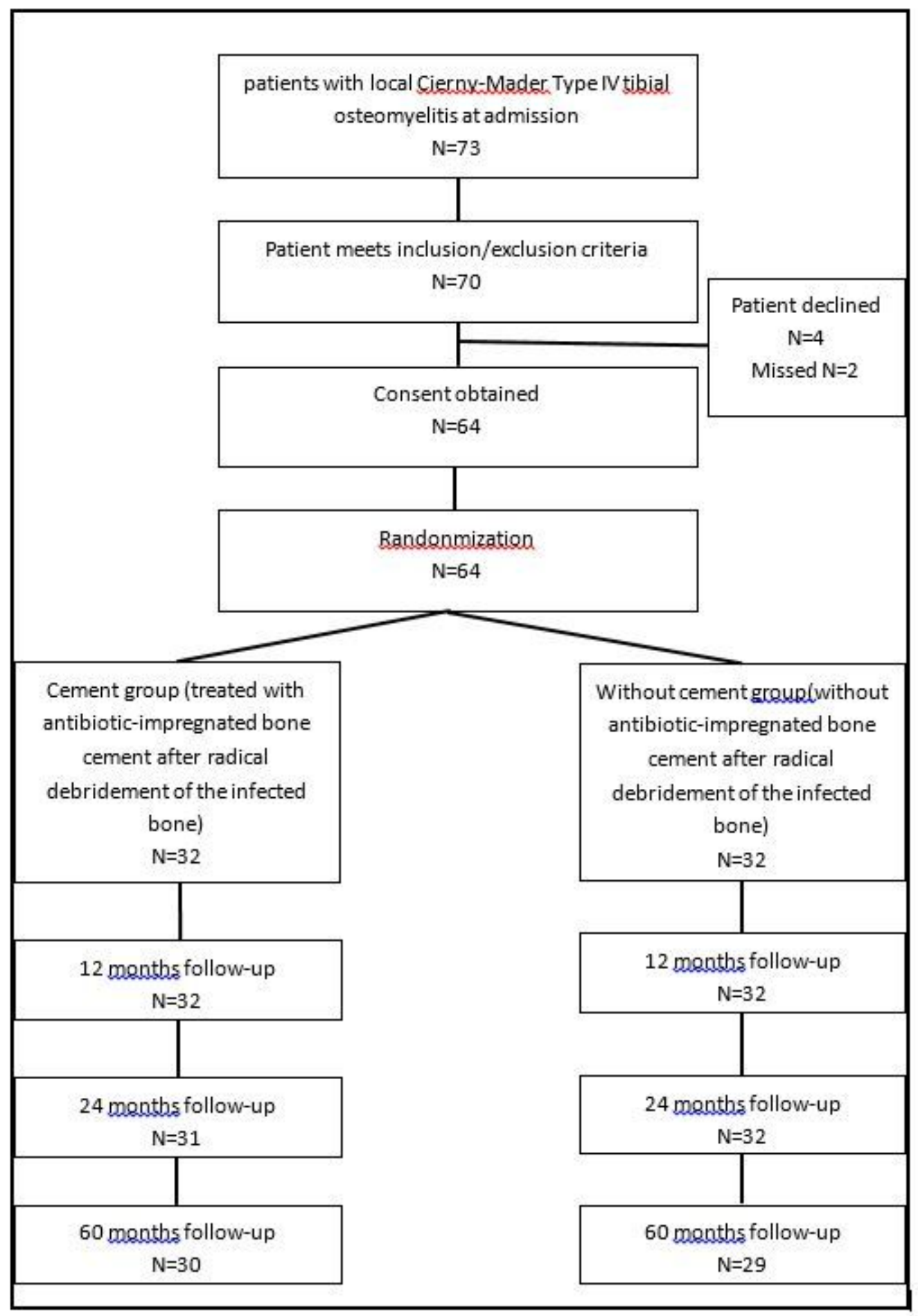

Figure 1

Flow diagram demonstrating study design 


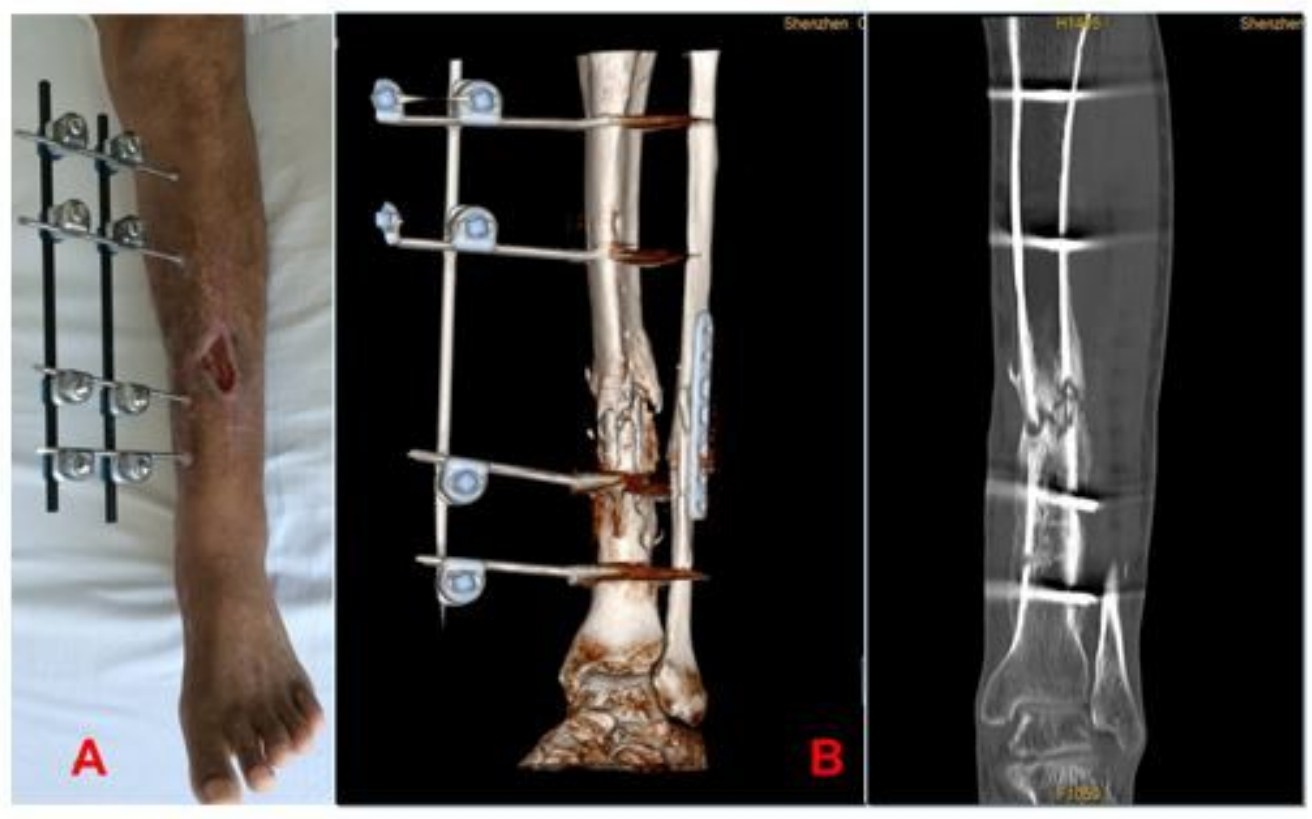

Figure 2

Male®39ys, infection 3 months after open reduction and internal fixation of left tibial, 4 months after replacement of external fixator ( A Clinical view; B CT scan)

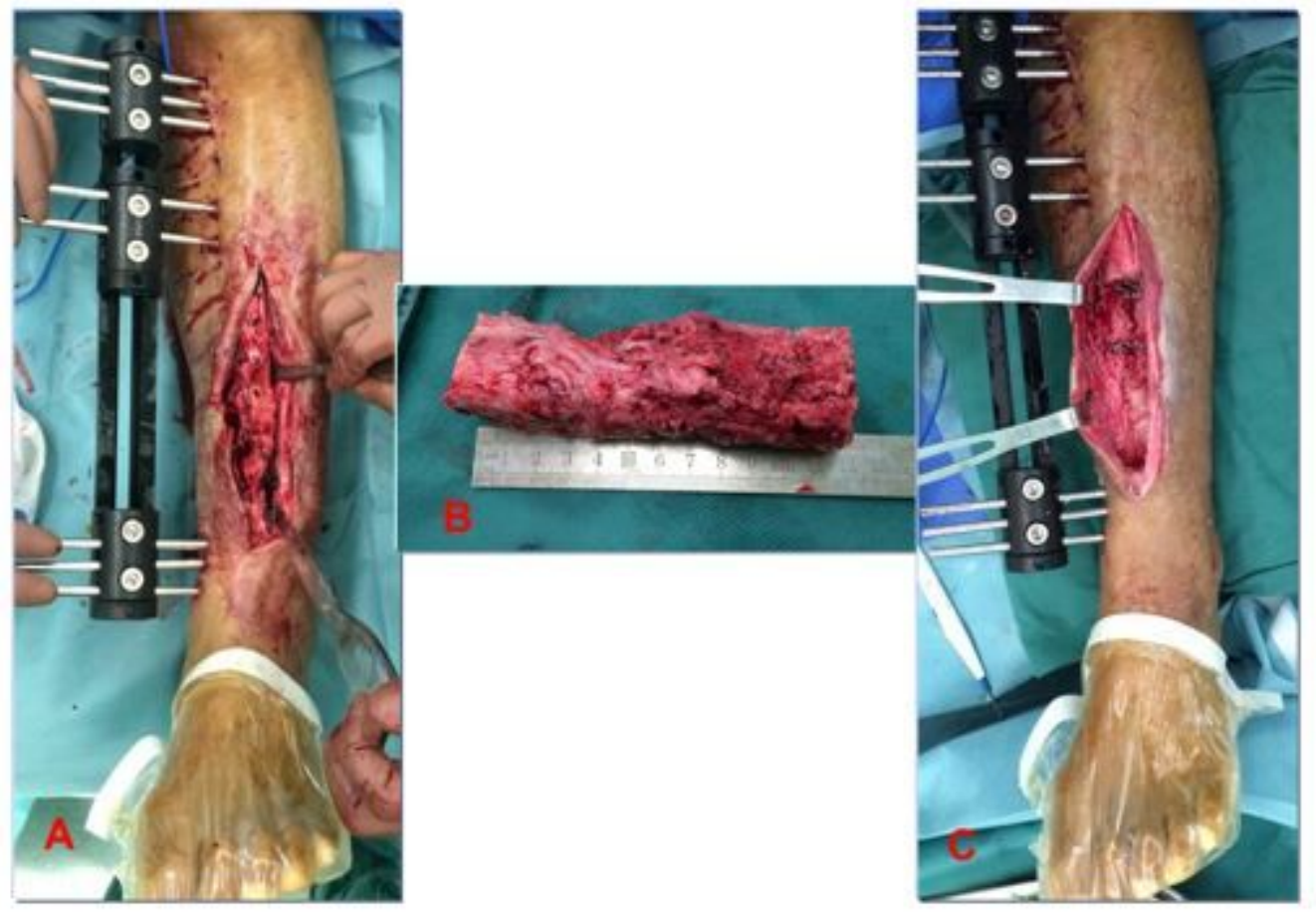

\section{Figure 3}

Radical debridement (A Appearance view of bone infection; B Infected bone segment; C Appearance view after debridement, bone defect: $12.8 \mathrm{~cm}$ ) 


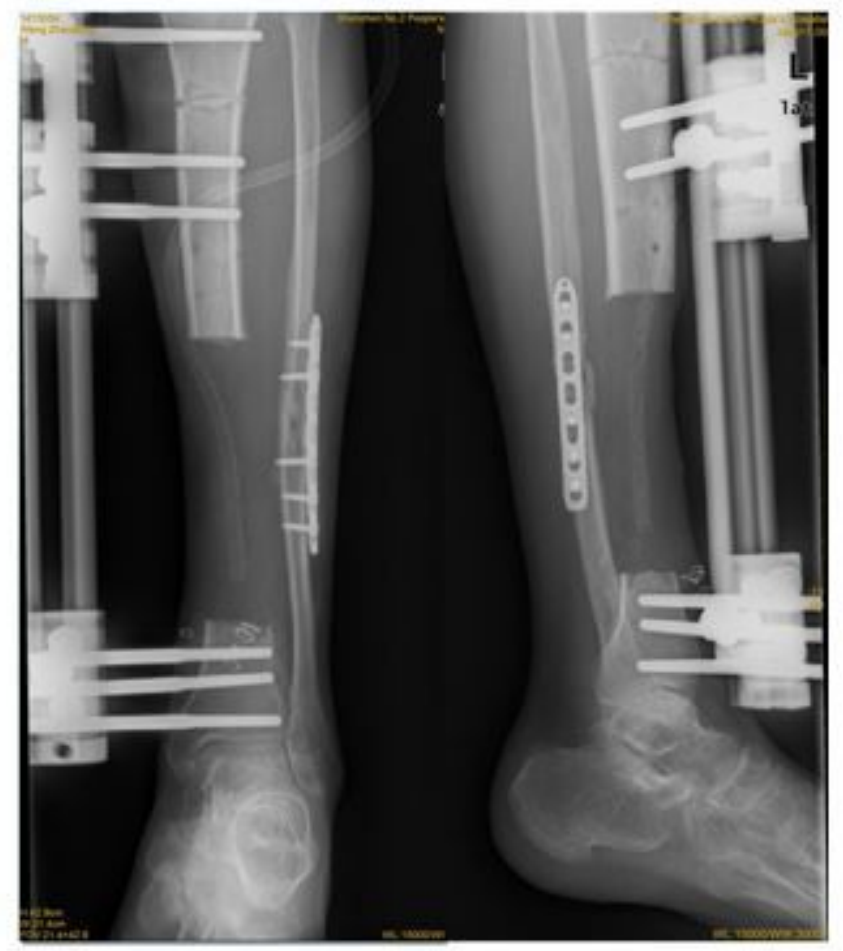

Figure 4

$X$ ray after radical debridement and replacement of LRS, without antibiotic cement

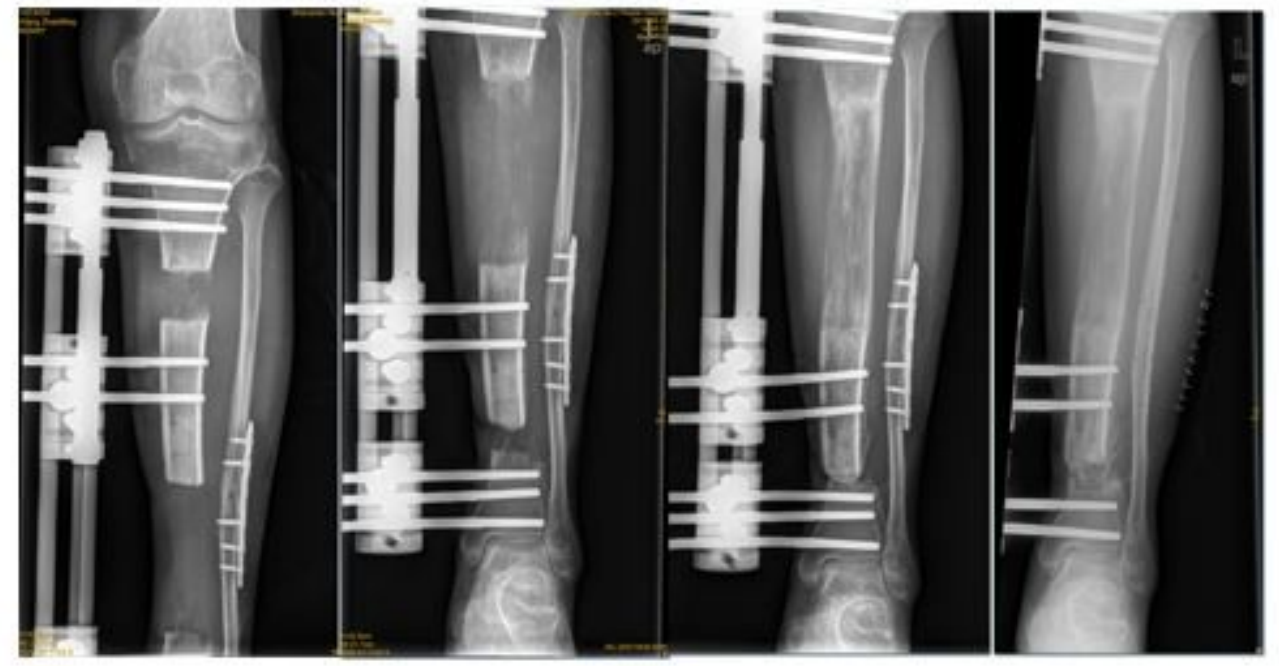

Figure 5

Bone transport and docking site grafting (removed the fibular plate at the same time) 


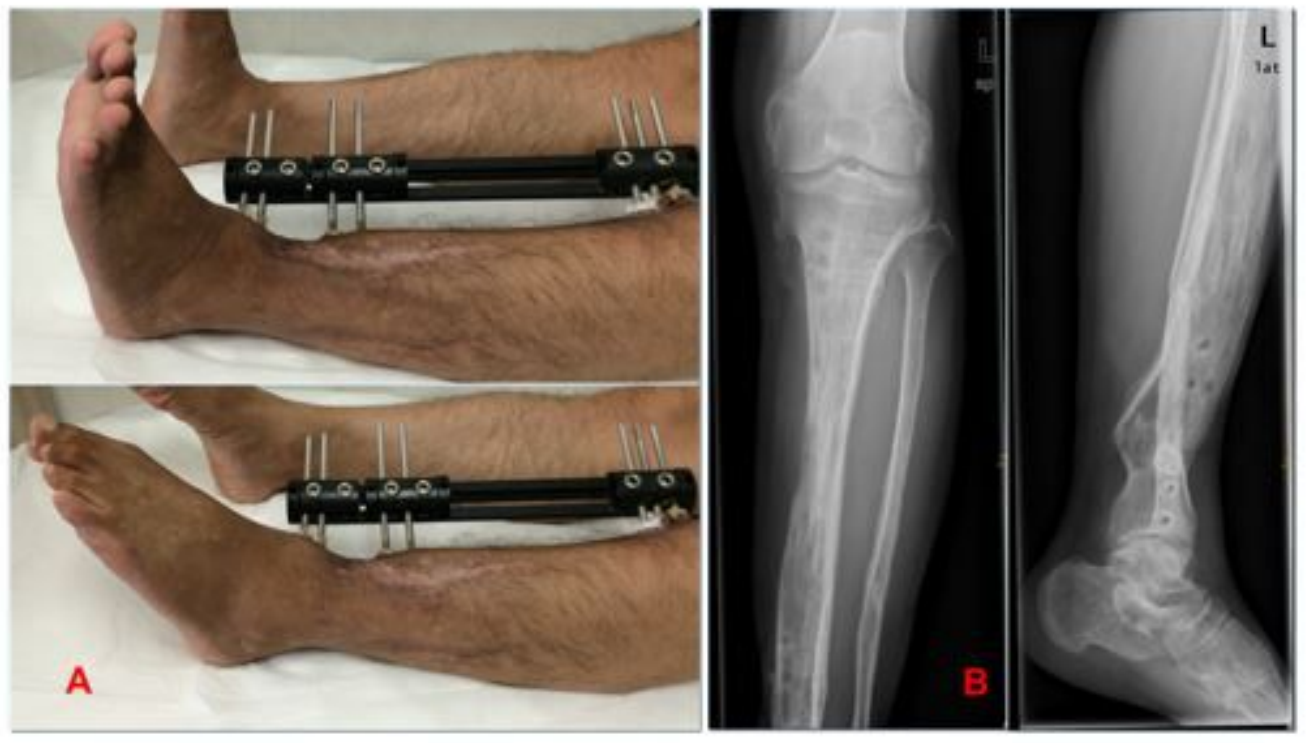

Figure 6

A Clinical appearance before the removal of LRS; B X ray after the removal of LRS.

\section{Supplementary Files}

This is a list of supplementary files associated with this preprint. Click to download.

- CONSORT2010Checklist.doc 